\title{
Crystal Structure and Synthesis of 6-(Phtalimidomethyldimethylsilanyl)- hexan-1-ol
}

\author{
Laurent LATXAGUE*广 and Jean-Michel LEGER ${ }^{* *}$ \\ *INSERM U577-Biomatériaux et Réparation Tissulaire, Université Victor Segalen \\ Bordeaux 2, 146 rue Léo Saignat, 33076 Bordeaux Cedex, France \\ **EA 2962-Pharmacochimie, Université Victor Segalen Bordeaux 2, 146, rue Léo Saignat, \\ 33076 Bordeaux, France
}

\begin{abstract}
A key intermediate in the synthesis of original organosilicon ascorbic acid derivatives, 6(phtalimidomethyldimethylsilanyl)hexan-1-ol crystallizes in a triclinic form, $P \overline{1}$. The unit-cell dimensions at $296 \mathrm{~K}$ are $a$ $=7.807(8), b=10.145(4), c=11.886(4) \AA, \alpha=93.53(3)^{\circ}, \beta=105.84(4)^{\circ}, \gamma=89.77(5)^{\circ}, V=903.8(10) \AA^{3}, D_{x}=1.174$ $\mathrm{g} / \mathrm{cm}^{3}$, and $Z=2$. The $R$ value is 0.0657 for 2662 observed reflexions.
\end{abstract}

(Received May 27, 2004; Accepted July 12, 2004; Published on web September 22, 2004)

As part of an ongoing study aimed at synthesizing organosilicon derivatives of $\mathrm{L}$-ascorbic acid as potential bone remodeling agents, ${ }^{1}$ we synthesized a series of silanol compounds 1a - d that can be equally considered as interesting building blocks for the synthesis of organosilicon bioactive molecules (Scheme 1). Thus, the hydrosilylation of unsaturated acetates $\mathbf{4 a}-\mathbf{d}$ with chloromethyldimethylsilane gave the saturated acetoxy derivatives 3a-d in $80 \%$ average yield. The latter compounds were subjected to a transesterification in methanol to afford the silyl alcohols $\mathbf{2 a}-\mathbf{d}$ in almost quantitative yields, and then reacted with potassium phtalimide to yield compounds $1 \mathbf{1 a}-\mathbf{d}$. Among them, 6-(phtalimidomethyldimethylsilanyl)hexan-1-ol $(\mathbf{1 d}, n=4)$ was isolated as an oil after silica-gel chromatography (hexane/ethyl acetate 6:4, 76\% yield). Single crystals for X-ray crystallography were prepared by slow evaporation from hexane/ethyl acetate (6:4) at room temperature.

Unfortunately, no other phtalimido derivatives (namely 1a-c) crystallized so easily, and we were not able to find a suitable solvent mixture to recrystallize them. Although crystalstructure data on hypervalent silicon derivatives (penta- or hexacoordinate complexes) are quite numerous (the subject was reviewed in $1986^{2}$ ) literature reports concerning X-ray determinations of simpler organosilicon compounds are sparse.

In this study, the crystal structure of compound 1d was

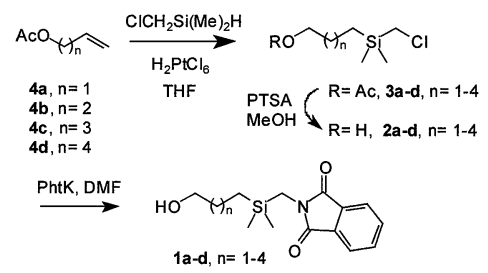

Scheme 1

† To whom correspondence should be addressed.

E-mail: laurent.latxague@u-bordeaux2.fr analyzed. ${ }^{3}$ Crystal and experimental data are given in Table 1.

Lorentz and polarization corrections and an empirical absorption correction were applied. The positions of non- $\mathrm{H}$ atoms were easily determined by the program SHELXS-97, and the positions of the $\mathrm{H}$ atoms were deduced from the coordinates of the non-H atoms and confirmed by Fourier synthesis. The non- $\mathrm{H}$ atoms were refined with anisotropic temperature

Table 1 Crystal and experimental data

Formula: $\mathrm{C}_{17} \mathrm{H}_{25} \mathrm{NO}_{3} \mathrm{Si}$

Formula weight: 319.47

Crystal system: triclinic $\quad Z=2$

Space group: $P \overline{1}$

$a=7.807(8) \AA$

$b=10.145(4) \AA$

$c=11.886(4) \AA$

$\alpha=93.53(3)^{\circ}$

$\beta=105.84(4)^{\circ}$

$\gamma=89.77(5)^{\circ}$

$V=903.8(10) \AA^{3}$

Colorless

Crystal size: $0.40 \times 0.30 \times 0.25 \mathrm{~mm}^{3}$

$D_{\mathrm{x}}=1.174 \mathrm{Mg} / \mathrm{m}^{3}$

$\mathrm{F}\left(\begin{array}{lll}0 & 0 & 0\end{array}\right)=344$

$\mu=1.239 \mathrm{~mm}^{-1}$

Temperature $=296(2) \mathrm{K}$

$\theta_{\max }=64.91^{\circ}$

Radiation: $\mathrm{Cu} K_{\alpha}(\lambda=1.54178 \AA)$

No. of reflections used: $2662[I>2 \sigma(I)]$

$R=0.0657$

$w R^{2}=0.1902$

Goodness-of-fit $=1.060$

$(\Delta / \sigma)_{\max }=0.012$

$(\Delta \rho)_{\max }=0.504 \AA^{-3}$

$(\Delta \rho)_{\min }=-0.370 \AA^{-3}$

Measurement: Enraf-Nonius CAD4 ${ }^{4}$

Structure determination: SHELXS-97 ${ }^{5}$

Refinement: SHELXL-976 
Table 2 Final atomic coordinates and equivalent isotropic thermal parameters $\left(U_{\text {eq }}\left(\AA^{2}\right)\right)$

\begin{tabular}{lllll}
\hline Atom & \multicolumn{1}{c}{$y$} & \multicolumn{1}{c}{$z$} & $U_{\text {eq }}$ \\
\hline $\mathrm{C}(1)$ & $1.1716(3)$ & $0.1225(2)$ & $0.1168(2)$ & $0.052(1)$ \\
$\mathrm{C}(2)$ & $1.0567(3)$ & $0.0478(2)$ & $0.1578(2)$ & $0.051(1)$ \\
$\mathrm{C}(3)$ & $1.0968(4)$ & $-0.0792(3)$ & $0.1898(3)$ & $0.065(1)$ \\
$\mathrm{C}(4)$ & $1.2579(4)$ & $-0.1290(3)$ & $0.1787(3)$ & $0.073(1)$ \\
$\mathrm{C}(5)$ & $1.3729(4)$ & $-0.0536(3)$ & $0.1382(3)$ & $0.071(1)$ \\
$\mathrm{C}(6)$ & $1.3313(3)$ & $0.0735(3)$ & $0.1068(3)$ & $0.065(1)$ \\
$\mathrm{C}(7)$ & $1.0896(4)$ & $0.2528(3)$ & $0.0900(2)$ & $0.058(1)$ \\
$\mathrm{N}(8)$ & $0.9275(3)$ & $0.2496(2)$ & $0.1176(2)$ & $0.055(1)$ \\
$\mathrm{C}(9)$ & $0.8981(3)$ & $0.1288(3)$ & $0.1569(2)$ & $0.056(1)$ \\
$\mathrm{O}(10)$ & $1.1455(3)$ & $0.3460(2)$ & $0.0509(2)$ & $0.087(1)$ \\
$\mathrm{O}(11)$ & $0.7646(3)$ & $0.0987(2)$ & $0.1840(2)$ & $0.082(1)$ \\
$\mathrm{C}(12)$ & $0.7974(4)$ & $0.3561(3)$ & $0.0960(2)$ & $0.064(1)$ \\
$\mathrm{Si}(13)$ & $0.8157(1)$ & $0.4846(1)$ & $0.2208(1)$ & $0.067(1)$ \\
$\mathrm{C}(14)$ & $0.7795(5)$ & $0.4098(3)$ & $0.3512(3)$ & $0.080(1)$ \\
$\mathrm{C}(15)$ & $0.5960(5)$ & $0.3544(4)$ & $0.3383(3)$ & $0.087(1)$ \\
$\mathrm{C}(16)$ & $0.5711(6)$ & $0.2952(4)$ & $0.4464(3)$ & $0.098(1)$ \\
$\mathrm{C}(17)$ & $0.3940(6)$ & $0.2329(5)$ & $0.4322(3)$ & $0.102(1)$ \\
$\mathrm{C}(18)$ & $0.3666(8)$ & $0.1771(6)$ & $0.5401(4)$ & $0.140(2)$ \\
$\mathrm{C}(19)$ & $0.1862(12)$ & $0.1189(8)$ & $0.5256(6)$ & $0.209(4)$ \\
$\mathrm{O}(20)$ & $0.1679(12)$ & $0.0777(17)$ & $0.6217(9)$ & $0.367(6)$ \\
$\mathrm{C}(21)$ & $0.6423(6)$ & $0.6075(4)$ & $0.1627(3)$ & $0.097(1)$ \\
$\mathrm{C}(22)$ & $1.0419(5)$ & $0.5604(4)$ & $0.2584(4)$ & $0.092(1)$ \\
\hline
\end{tabular}

$U_{\mathrm{eq}}=(1 / 3) \Sigma_{i} \Sigma_{j} U_{i j}\left(a_{i}^{*} a_{j}^{*}\right)\left(\boldsymbol{a}_{i} \cdot \boldsymbol{a}_{j}\right)$.

Table 3 Selected torsion angles $\left({ }^{\circ}\right)$

$\begin{array}{lc}\mathrm{Si}(13)-\mathrm{C}(14)-\mathrm{C}(15)-\mathrm{C}(16) & -179.6(3) \\ \mathrm{C}(14)-\mathrm{C}(15)-\mathrm{C}(16)-\mathrm{C}(17) & -176.3(4) \\ \mathrm{C}(15)-\mathrm{C}(16)-\mathrm{C}(17)-\mathrm{C}(18) & -178.4(4) \\ \mathrm{C}(16)-\mathrm{C}(17)-\mathrm{C}(18)-\mathrm{C}(19) & 178.1(6) \\ \mathrm{C}(17)-\mathrm{C}(18)-\mathrm{C}(19)-\mathrm{O}(20) & -177.3(10)\end{array}$

Table 4 Selected bond distances $\left({ }^{\circ}\right)$

\begin{tabular}{ll}
\hline $\mathrm{Si}(13)-\mathrm{C}(12)$ & $1.889(3)$ \\
$\mathrm{Si}(13)-\mathrm{C}(14)$ & $1.855(4)$ \\
$\mathrm{Si}(13)-\mathrm{C}(21)$ & $1.856(5)$ \\
$\mathrm{Si}(13)-\mathrm{C}(22)$ & $1.857(5)$ \\
\hline
\end{tabular}

Table 5 Hydrogen-bonding geometry $\left(\AA,{ }^{\circ}\right)$ and van der Waals contact $(\AA)$

\begin{tabular}{|c|c|}
\hline $\mathrm{O} 20 \ldots \mathrm{O} 11(\mathrm{I})$ & $2.941(14)$ \\
\hline $\mathrm{H} 20 \ldots \mathrm{O} 11(\mathrm{I})$ & 2.574 \\
\hline $\mathrm{O} 20-\mathrm{H} 20 \ldots \mathrm{O} 11(\mathrm{I})$ & 108.6 \\
\hline $\mathrm{C} 21 \ldots \mathrm{O} 10(\mathrm{II})$ & $3.445(6)$ \\
\hline H21... 10 (II) & 2.582 \\
\hline $\mathrm{C} 21-\mathrm{H} 21 \ldots \mathrm{O} 10(\mathrm{II})$ & 149.5 \\
\hline O11...C5(III) & $3.326(5)$ \\
\hline O11...C6(III) & $3.261(5)$ \\
\hline O20...C19(IV) & $3.414(16)$ \\
\hline $\mathrm{C} 3 \ldots \mathrm{C} 7(\mathrm{~V})$ & $3.597(6)$ \\
\hline C5...O11(VI) & $3.326(5)$ \\
\hline C6...O11(VI) & $3.261(5)$ \\
\hline $\mathrm{C} 7 \ldots \mathrm{C} 3(\mathrm{~V})$ & $3.597(6)$ \\
\hline C19...O20(IV) & $3.414(16)$ \\
\hline
\end{tabular}

Symmetry codes: I $(1-x,-y, 1-z)$; II $(2-x, 1-y,-z)$; III $(x-1, y, z)$; IV $(-x,-y, 1-z) ; \mathrm{V}(2-x,-y,-z)$; VI $(1-x,-y,-z)$.

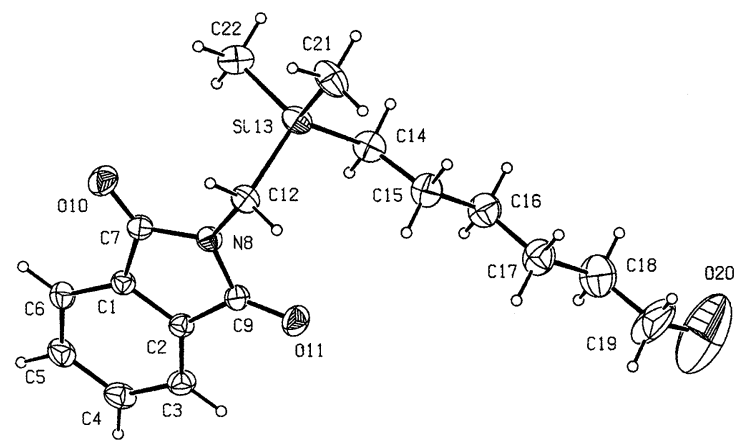

Fig. 1 Molecular structure with the numbering scheme. Displacement ellipsoids are drawn at the $30 \%$ probability level.

parameters. $\mathrm{H}$ atoms were inserted in calculated positions and not refined.

The structure of 6-(pthalimidomethyldimethylsilanyl)hexan-1ol is illustrated in Fig. 1. It shows an open carbon chain as evidenced by the torsion angle values around $180^{\circ}$ (Table 3 ). It must be noted that the silicon-carbon bond distances are in good agreement with the literature data $(d=1.857 \AA \text { for } \mathrm{C}-\mathrm{Si})^{7}$ (Table 4).

The aromatic phtalimido moiety is quasi planar, as expected, with least-squares plane variations of 0.003 and $0.008 \AA$ for the six and five-membered rings, respectively. The interplanar angle of the aromatic ring and the hexanol open chain is $65.0(4)^{\circ}$

Additionally, intermolecular stacking is provided by two hydrogen bonds and several van der Waals contacts (Table 5).

\section{References}

1. G. Vilaça, C. Rubio, J. Susperregui, L. Latxague, and G. Déléris, Tetrahedron, 2002, 58, 9249.

2. M. G. Voronkov and N. V. Alekseev, Top. Curr. Chem., 1986, 131, 99.

3. Full crystallographic results have been deposited at the Cambridge Crystallographic Data Centre (CCDC), UK, as Supplementary Material (deposition number CCDC 219221).

4. Enraf-Nonius CAD-4 Software, Version 5.0, 1989, EnrafNonius: Delft, The Netherlands.

5. G. M. Sheldrick, SHELXS 97, Program Crystal Structure Solution, 1997, University of Göttingen, Germany.

6. G. M. Sheldrick, SHELXL 97, Program for Crystal Structure Refinement, 1997, University of Göttingen, Germany.

7. F. H. Allen, O. Kennard, D. G. Watson, L. Brammer, A. G. Orpen, and R. Taylor, J. Chem. Soc. Perkin Trans. II, 1987, S1. 